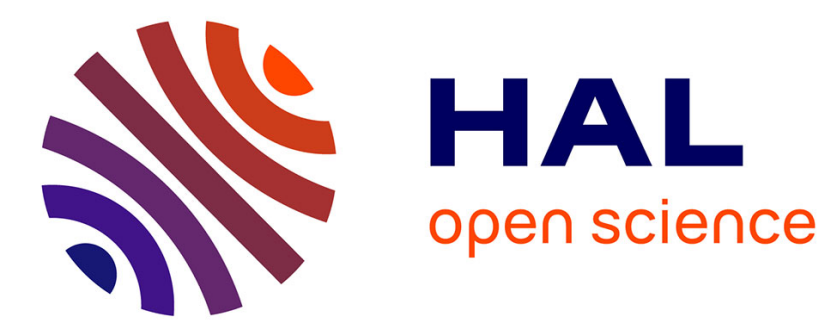

\title{
Un cas d'école pour les services d'archives, quand médiation et dispositif numérique éloignent le document
}

\author{
Jessica de Bideran
}

\section{To cite this version:}

Jessica de Bideran. Un cas d'école pour les services d'archives, quand médiation et dispositif numérique éloignent le document . Les Enjeux de l'information et de la communication, 2015. hal-01399488

\section{HAL Id: hal-01399488 \\ https://hal.science/hal-01399488}

Submitted on 18 Nov 2016

HAL is a multi-disciplinary open access archive for the deposit and dissemination of scientific research documents, whether they are published or not. The documents may come from teaching and research institutions in France or abroad, or from public or private research centers.
L'archive ouverte pluridisciplinaire HAL, est destinée au dépôt et à la diffusion de documents scientifiques de niveau recherche, publiés ou non, émanant des établissements d'enseignement et de recherche français ou étrangers, des laboratoires publics ou privés.

\section{(ㅇ)(1) $\$$}

Distributed under a Creative Commons Attribution - NonCommercial - NoDerivatives| 4.0 


\title{
Un cas d'école pour les services d'archives, quand médiation et dispositif numérique éloignent le document
}

\author{
A textbook case for departments of archives, when mediation \\ and digital device take away the document \\ Un caso de escuela para los servicios de archivos, cuando mediación \\ y dispositivo numérico alejan el documento
}

Article inédit, mis en ligne le 26 octobre 2015.

Jessica de Bideran

Jessica de Bideran est actuellement ingénieur de recherche à la Maison des Sciences de l'Homme d'Aquitaine et travaille sur l'édition numérique d'un patrimoine documentaire régional. Ces recherches portent plus généralement sur les enjeux de la numérisation du patrimoine et son impact sur les pratiques et systèmes de médiation créés en contexte de valorisation. Collaborateur permanent du laboratoire MICS LERASS de Toulouse, elle est également membre de l'Observatoire des mondes numériques en sciences humaines. Jessica de Bideran, MSHA, Université Bordeaux Montaigne, 33000 Bordeaux, France. jessica.debideran@gmail.com

\section{Plan}

Introduction

L'éducation artistique et culturelle, un lieu de valorisation

Un complexe équilibre entre valorisation de l'institution et valorisation du fonds

Entre description du document et organisation de l'archive, une appropriation complexe

Une médiation documentaire qui met à distance le document

Conclusion

Références bibliographiques

\section{Résumé}

Pour les institutions culturelles conservant un patrimoine documentaire, la numérisation soulève des enjeux importants de description, de circulation et d'appropriation de ces ressources. Médiatiser les documents numérisés suppose notamment de créer des écosystèmes de connaissances simples à exploiter et correspondant aux pratiques des différents usagers visés. L'article qui suit se concentre sur l'analyse détaillée d'un programme de valorisation numérique de documents iconographiques porté par les Archives municipales de Bordeaux. Cet exemple montre combien les logiques d'organisation documentaire des professionnels influent sur la création du dispositif numérique et mettent finalement à distance le document, pourtant au centre des missions de conservation et de diffusion des archives.

\section{Mots-clés}

Document, médiation, valorisation, dispositif numérique, archives. 


\begin{abstract}
For the cultural institutions preserving a documentary heritage, the digitalization raises important challenge in description, in circulation and in appropriation of these resources. Mediatize digital documents supposes in particular to create ecosystems of knowledge simple to exploit and correspondent in the practices of the various aimed users. This article which follows concentrates on the detailed analysis of a program of digital valuation of iconographic documents worn by the municipal Archives of Bordeaux. This example shows how much the logics of documentary organization of the professionals influence the creation of the digital device and put finally remotely the document, nevertheless in the center of the missions of preservation and distribution of archives.
\end{abstract}

\title{
Keywords
}

Document, mediation, valuation, digital device, archival.

\section{Resumen}

Para las instituciones culturales que conservan un patrimonio documental, la digitalización indigna puestas importantes de descripción, de circulación y de apropiación de estos recursos. Mediatizar los documentos digitalizados supone particularmente de crear ecosistemas de conocimientos simples que hay que explotar y corresponsal a las prácticas de los diferentes usuarios aludidos. El artículo que sigue se concentra sobre el análisis detallado de un programa de valorización numérica de documentos iconográficos llevado por los Archivos municipales de Burdeos. Este ejemplo muestra cuánto las lógicas de organización documental de los profesionales influyen en la creación del dispositivo numérico y finalmente ponen en distancia el documento, sin embargo en el centro de las misiones de conservación y de difusión de los archivos.

\section{Palabras clave}

Documento, mediación, valorización, dispositivo numérico, archivo.

\section{Introduction}

Transformant l'archive, document d'histoire, en objet culturel et trivial (Jeanneret 2008), la numérisation de masse du patrimoine documentaire promue par les pouvoirs publics soulève des enjeux importants de description, de circulation et d'appropriation de ces ressources. D'abord débattue sous l'angle économique et législatif, suite au bras de fer qui opposa en 2009 la BnF et Google (Dufrêne et al., 2013), la numérisation du patrimoine écrit interroge les professionnels de la documentation et engendre des modifications juridiques, techniques et organisationnelles (BrunPicard et Lallich-Boidin, 2011) dont les impacts sont encore difficiles à cerner. Face à cette injonction d'ouverture et d'accessibilité, il incombe en effet aux institutions culturelles détentrices de fonds numérisés de créer des écosystèmes de connaissances riches et intuitifs tout en visant des usagers dont les pratiques demeurent complexes à saisir. Or, la réalisation de ces outils de 
consultation documentaire suppose de réunir autour d'un objectif commun, une pluralité d'acteurs dont les logiques professionnelles parfois divergent. Cette "polyphonie patrimoniale " (Both et Garcia, 2014) n'est pas sans conséquence sur la circulation de l'objet document. Support transmettant de l'information, la ressource archivistique condense une matérialité tangible et une virtualité de sens qui se voit actualisée lors de sa réception par le lecteur. Or, si la reproduction digitale annule cette "expérience auratique» de l'archive (Klein et Lemay, 2014), le substitut numérique n'en est pas moins le véhicule d'une expérience cognitive qui s'appuie sur une spatialisation des connaissances via l'écran de consultation. Les mises en scène numériques du substitut influencent dès lors fortement l'activité de lecture qui se voit encadrée par le dispositif, et, conséquemment, par les professionnels qui le construisent.

Dans quelle mesure les logiques métiers des différents experts impliqués influent-elles sur l'architecture du dispositif ? L'archive, en tant que matériau, étant à l'origine de ces projets de valorisation numérique, en quoi les différentes dimensions assignées au document par ces professionnels entrent-elles en concurrence ? À travers l'article qui suit nous souhaitons apporter des éléments de réponse à ces questions en nous concentrant sur l'analyse détaillée d'un programme de numérisation et de valorisation de documents iconographiques porté par les Archives municipales de la ville de Bordeaux. Ce travail s'appuie sur une expertise menée sous l'égide de la Direction Régionale des Affaires Culturelles d'Aquitaine qui participe au financement de ce programme via le programme régional Banque numérique du savoir en Aquitaine (Sibers, 2011); il s'agissait plus particulièrement d'établir des préconisations visant à étendre ce dispositif à un plus grand nombre de classes. Réalisée entre septembre 2013 et juin 2014 quatre classes engagées dans ce projet pédagogique intitulé «Cas d'écoles - Cyprien Alfred-Duprat - Éduquer autrement au patrimoine et à l'architecture" ont été suivies. L'étude documentaire des ressources constituant le corpus d'archives; l'analyse sémio-pragmatique du dispositif numérique proposé aux enseignants ; des entretiens libres menés avec le groupe de travail de l'académie de Bordeaux ainsi qu'avec les différents enseignants ayant expérimenté ce système; une série d'observations distanciées des pratiques exploitées par les enseignants; autant d'examens qui construisent un terrain d'étude hybride ${ }^{1}$. Celui-ci facilite selon nous la compréhension des enjeux culturels et professionnels qui accompagnent la création de cette action, en soulevant notamment les points de blocage ou les stratégies des différents acteurs. Élaboré dans le cadre l'Éducation artistique et culturelle, ce système donne en effet à voir les multiples appropriations auxquelles est aujourd'hui soumis le patrimoine documentaire. Accélérées par le numérique, ces tentatives d'assimilation brouillent le concept de médiation qui paraît plus que jamais difficile à définir. Action permettant d'établir un lien entre un objet complexe et des sujets volontaires, la médiation suppose une appropriation informationnelle par un dévoilement du sens. Les politiques d'animation culturelle et le développement de services pédagogiques au sein des institutions ont renforcé la diffusion de l'expression « médiation culturelle " pour nommer des activités aussi diverses que les visites guidées ou les expériences en ateliers (Chaumier et Mairesse, 2013). Par ailleurs, les professionnels de la documentation nomment "médiation documentaire " l'action de créer des documents secondaires traduisant des documents primaires, à l'image des catalogues ou des cartels d'œuvres dans un musée. Or, avec le numérique, les dispositifs faisant fonction d'inventaire informationnel et d'outil communicationnel tendent à se confondre, si bien qu'il paraît opportun de définir ces systèmes comme des documents partagés

\section{$\ldots \ldots$}

'Les classes ont pu être suivies à plusieurs reprises entre septembre 2013 et juin 2014 mais les entretiens et la rédaction proprement dite du rapport se sont déroulés entre mars et juin 2014. Pour les entretiens libres, au total, 10 personnes ont pu être interrogées; les citations entre guillemets et suivies des initiales entre crochets sont extraites de ces entretiens. Le faible nombre de personnes impliquées n'a pu cependant permettre la mise en place d'analyses statistiques plus fines. 
(Régimbeau, 2014: 46). Toutefois, diffuser des archives ne signifie pas pour autant médiatiser les informations historiques dont ces dernières sont porteuses. De fait, nous forgeons l'hypothèse qu'en hybridant les processus de médiation, puisque médiation culturelle et médiation documentaire convergent ici en une action pédagogique complexe, ces politiques de valorisation éloignent en définitive la dimension documentaire de l'archive qui devient l'objet d'appropriations multiples et parfois concurrentes.

\section{L'éducation artistique et culturelle, un lieu de valorisation}

En quelques années, les politiques de valorisation menées par les services d'archives ont été profondément bouleversées par les potentialités offertes par les outils numériques tout autant que par l'impératif communicationnel exigé par les pouvoirs publics. Longtemps centrés sur l'accueil en salle de lecture d'historiens ou de généalogistes, ces établissements ont depuis appréhendé d'autres formes de présentation des ressources conservées en créant, par exemple, des expositions réelles et virtuelles ou en développant des ateliers pédagogiques destinés aux scolaires (Ciosi, 2013). À cet égard, les différents programmes de numérisation lancés ces dernières années par les Archives municipales de la ville de Bordeaux illustrent parfaitement cette optique d'ouverture et d'accessibilité (Vatican, 2011). En s'appuyant sur ses fonds et sur les technologies de l'information et de la communication, l'institution a notamment développé, avec l'aide des services culturels de la ville, une série d'animations destinées aux écoles élémentaires équipées de tableaux numériques interactifs dans le but de sensibiliser ces jeunes publics à l'architecture et au patrimoine de leur cité. Se déroulant sur la durée de l'année scolaire, le programme "Cas d'écoles - Cyprien Alfred-Duprat» débute avec la sélection des quatre classes dont les enseignants ont répondu à l'appel à projet pédagogique lancé au mois de juin précédent par l'Académie de Bordeaux. Ces derniers suivent alors une demi-journée de formation assurée par les conseillers pédagogiques du rectorat. Ceux-ci leur exposent le projet dans sa globalité, c'est-à-dire le corpus numérisé, les intervenants que sont les archivistes et animateurs du patrimoine qui les accompagnent tout au long de l'année, les sujets susceptibles d'être traités à partir du corpus avec les élèves et les attendus en termes de restitution artistique.

Selon la convention entérinant administrativement le projet, il s'agit pour les jeunes apprenants de s'inscrire dans un parcours culturel autour du patrimoine architectural de Bordeaux tout en s'engageant dans l'utilisation raisonnée de l'informatique pour s'informer, se documenter et communiquer des savoirs. Initiation au numérique et initiation au patrimoine sont donc les deux objectifs qui sous-tendent ce volet pédagogique. Proposé aux enseignants à partir de l'année scolaire 2012-2013, le programme s'est poursuivi en 2013-2014 et huit classes ont pu à ce jour bénéficier de cette offre". Contrairement à ce qui était souhaité en 2012 lors du lancement de "Cas d'école ", le programme peine donc à s'étendre à l'ensemble des classes du cycle 3 de Bordeaux. Cette situation tient, nous semble-t-il, au processus de communication retenu par la municipalité. Cherchant à initier les élèves à la découverte patrimoniale dans un cadre plus souple que les cours, le programme entretient notamment une certaine ambiguité entre activité de médiation, qui suppose échanges et dialogues entre les différents acteurs en présence dans un temps et un lieu donnés, et système de

\footnotetext{
² Le programme n'a pu être proposé aux enseignants durant l'année scolaire 2014-2015 car les Archives municipales de Bordeaux changent de site et déménagent dans des locaux plus adaptés au nombre croissant des publics à accueillir. L’année 2015 est donc consacrée à la préparation et au transfert des fonds.
} 
valorisation qui vise essentiellement à mettre en scène l'institution et ses ressources numérisées. Le traitement documentaire du corpus puis sa diffusion sur support médiatique, deux étapes clés de la valorisation numérique (Casemajor-Lousteau, 2013) assurées par le personnel des Archives, sont ainsi associés à des pratiques de médiation culturelle plus traditionnelles telles que la visite guidée réalisée par une animatrice du patrimoine. Ce contexte d'hybridation, s'il présente des avantages sur lesquels nous reviendrons, n'est pas sans entraîner une certaine confusion sur les orientations et donc sur l’intelligibilité du programme.

\section{Un complexe équilibre entre valorisation de l'institution et valorisation du fonds}

Ainsi, le départ officiel du projet est donné au cours du premier trimestre par la visite pour chaque classe des Archives municipales. Celle-ci se déroule en demi-groupe, la classe étant scindée en deux du fait des difficultés de circulation à l'intérieur du site. Les Archives bordelaises sont en effet installées depuis 1939 au sein de l'ancien hôtel particulier Ragueneau. Or, cet édifice classé Monument Historique en 1964 s'avère de moins en moins adapté à l'accueil des publics et à la conservation des fonds qui, tous deux, ne cessent de croître. De fait, pendant qu'un animateur du patrimoine donne à voir l'architecture de l'hôtel de Ragueneau et délivre des éléments de compréhension de ce monument du XVII siècle, le second groupe visite les Archives et rencontre un professionnel qui présente l'activité de ce service administratif. Au-delà de la découverte de ce secteur patrimonial, généralement inconnu des élèves, ce moment est l'occasion de se confronter aux documents originaux dans leur temporalité et leur matérialité, documents qu'ils n'ont jusqu'à présent consultés que sous format numérique. Si cette rencontre avec le document permet de lui donner une authenticité, phénomène que ne peut assurer sa présentation numérique, la visite guidée du monument parait saugrenue aux enseignants qui peinent à expliquer à leurs élèves le lien entre ce moment d'initiation architecturale avec la révélation des ressources archivistiques originales. L'intérêt de cette séquence, quelque peu incongrue pour ses destinataires, est pourtant fermement soutenu par le personnel des Archives qui ne peut concevoir la mise en œeuvre de ce projet sans cet accueil sur site des jeunes scolaires. Certes, la présentation des documents originaux permet de rétablir l'épaisseur du temps, les élèves expérimentant ainsi leurs dimensions, la matière et la richesse de leurs couleurs, mais la visite guidée assure surtout pour le service des Archives la venue de groupes scolaires. Pour diverses raisons qui tiennent à sa reconnaissance au sein de la collectivité, il est en effet aujourd'hui primordial pour cette structure de promouvoir ses activités et l'importance de ses missions au-delà du cercle fermé des historiens et férus de généalogie. In fine, il s'agit pour les Archives de capter un jeune public qui, depuis les politiques de démocratisation culturelle initiées dans les années 1980 par les musées se concentre plus traditionnellement dans les salles de ces établissements. Quitte à entretenir la confusion entre la valorisation de l'institution Archives et la valorisation du fonds d'archives...

La seconde intervention de l'animateur du patrimoine est quant à elle organisée au début du second trimestre et consiste en une nouvelle visite guidée. Les classes arpentent en quelques heures la Maison Cantonale, seule réalisation architecturale véritablement aboutie de Cyprien Alfred-Duprat. Architecte urbaniste visionnaire, Cyprien Alfred-Duprat (1876-1933) est en effet un personnage original, utopiste, passionné par la modernité et ses avatars tels que l'aéronautique ou l'automobile, 
mais dont les créations restèrent pour leur grande majorité à l'état de projet. ${ }^{3}$. Cette animation est donc mieux appréhendée par les élèves et enseignants qui perçoivent aisément et rapidement le lien entre les illustrations vues en classe et la découverte de cet édifice dont ils analysent, à l'aide de photocopies distribuée sur place, la représentation dressée par l'architecte en 1925. Dans la continuité de cette initiation architecturale, ici ce sont les qualités artistiques de l'archive qui sont mises en avant par le médiateur qui délaisse volontairement les dimensions documentaire et historique de la ressource. En présentant à ce jeune public avant tout une "belle image " [ILA] imprimée en grand format et en couleurs, cette professionnelle de la médiation, en l'occurrence historienne de l'art, construit une relation à l'archive essentiellement sensible ; à cet égard, les élèves sont invités à dessiner sur la feuille qui leur est distribuée. La figure de l'auteur de l'image, l'architecte Cyprien-Alfred Duprat, l'esthétique et l'originalité de son travail sont surqualifiées dans le discours de l'animatrice. L'image présentée est confrontée au monument; elle devient l'objet d'une déconstruction iconographique organisée à partir des questions des élèves. Or, si le rapport au monument est parfaitement construit, conformément à la pratique traditionnelle de cette animatrice habituée à organiser des visites guidées architecturales, le rapport à l'ensemble du corpus numérisé est totalement absent de cette séquence puisque qu'aucun support, analogique ou numérique, ne permet de replacer l'illustration de la Maison Cantonale dans cet ensemble. Engagée dans une pratique de médiation monumentale qu'elle maitrise, rompue à l'exercice par des années d'accompagnement de groupes en visite, l'animatrice ne renvoie donc à aucun moment au reste du corpus documentaire. De fait, celle-ci se considère "exclue du dispositif numérique sur lequel travaillent les enseignants et élèves puisqu'aucune intervention en classe n'est prévue" [ILA]. À l'inverse de cette expérience sensible de communication analogique donc, les conditions de lecture proposées par les archivistes via le dispositif numérique s'appuient essentiellement sur une pratique professionnelle d'organisation documentaire et de description informationnelle. La stratégie actuellement mise en ouvre hybride en effet une forme de valorisation patrimoniale traditionnelle, autrement dit la découverte d'un site historique par le truchement d'une médiation présentielle, avec une exploitation de substituts numériques qui sont pour leur part organisés selon un système de valorisation plus organisationnelle que sensible.

\section{Entre description du document et organisation de l'archive, une appropriation complexe}

Le corpus recouvre une sélection de 20 planches numérisées en haute définition à partir du fonds Cyprien Alfred-Duprat conservé par les Archives qui comprend dans son ensemble 98 dessins. Transmis aux enseignants sous la forme d'un DVD-Rom gravé par les archivistes, le dispositif de consultation est organisé selon une architecture hypertextuelle de type HTML. Les enseignants

\footnotetext{
$\ldots \ldots$

L'ensemble de ces dessins fut réuni en 1929 lors de l'édition de l'ouvrage Bordeaux...un jour ! tiré seulement à 350 exemplaires. Cet ouvrage a récemment fait l'objet d'une réédition justement enrichie d'un CD-Rom. Cf. : Alfred-Duprat, Cyprien (2011): Bordeaux...un jour !, postface de Marc Saboya, Archives Municipales de Bordeaux et Société Archéologique, Bordeaux : Le festin.

'Les expressions entre guillemets et en italiques suivies d'initiales entre crochets sont extraites des entretiens libres menés avec les acteurs du projet lors de la phase d'étude entre mars et juin 2014.

Précisons qu’à l'heure actuelle, et suite à la refonte du site Internet des Archives municipales de Bordeaux qui ne possédaient pas jusqu'à présent d'espace numérique et de serveurs web dédiés, ces ressources sont aujourd'hui accessibles en ligne. La consultation du document se fait après sélection d'une vignette iconographique, système de visualisation qui correspond désormais à un standard de l'Internet, bien loin du dispositif que nous analysons ici.
} 
travaillent ensuite en classe via le tableau numérique interactif, tout au long de l'année et selon une organisation personnelle en séances pédagogiques. Une exploration longue et minutieuse du dispositif permet de dégager la façon dont les concepteurs ont projeté l'exploitation d'une telle édition numérique, conformément au principe même de l'interactivité (Le Marec, 1993). C'est donc par la description critique que l'on se propose d'aborder ce corpus d'archives outillé, l'angle privilégié étant celui de l'appropriation et de la pratique de l'outil. Cette description est renforcée par un recours à la sémiotique des écrits d'écran afin d'analyser les propriétés de l'outil, son statut éditorial et communicationnel ainsi que la structuration informationnelle qu'il propose (Jeanneret et Souchier, 2005). Ainsi les lignes qui suivent reprennent la progression que nous avons suivie à travers les agencements qui nous sont apparus essentiels ; l'identification du corpus et son appareil critique, les modalités de circulation dans la collection iconographique et les propriétés conférées à l'hypertextualité constituent autant d'éléments qui renseignent le chercheur sur les relations aux documents induites par le système de présentation numérique.

D'emblée le chercheur note la forte exposition de l'institution Archives sur l'écran d'accueil du dispositif qui présente brièvement la structure et qui est orné de plusieurs logos de l'établissement. De même, celui-ci précise que "l'instrument de recherche la étél produit au moyen du logiciel Arkhéia" [JCL] ; ce signalement n'est pas anecdotique car ce système de classement numérique produit par la société Anaphore est exclusivement réservé aux institutions conservant des archives. Peu souple, l'exploitation de cet outil s'est avérée extrêmement contraignante pour la planification du système de consultation. Le dispositif se présente en effet comme une longue page de texte divisée en chapitres que l'utilisateur peut faire entièrement défiler, soit grâce au système d'ascenseur caractéristique des interfaces de bureautique, soit en cliquant directement sur les titres des chapitres dans le pavé intitulé "Sommaire " et situé à gauche de l'écran (fig.1). Ces chapitres sont au nombre de quatre : "Présentation ", "Aide à l'enseignant ", "Corps de l'instrument de recherche " et "Index ». Le lien " Aide à l'enseignant » ouvre, dans une fenêtre supplémentaire, un document PDF de 48 pages rédigé par les acteurs du programme. Ce texte tient lieu tout à la fois de mode d'emploi de l'objet numérique et de dossier pédagogique proposant aux enseignants de les accompagner dans la découverte des ressources et leur exploitation en classe (description du sujet, questions qui peuvent être posées aux élèves, etc.).

Les témoignages recueillis montrent cependant que cette documentation est très peu exploitée par les enseignants qui la lisent une fois en début de projet - et donc d'année - mais qui n’y reviennent plus par la suite, même lorsque ces derniers sont "perdus face à l'ampleur du programme proprement-dit et n'ont pas su prendre l'investissement qu'il représente» [MPO]. Les modules "Corps de l'instrument de recherche » et "Index " sont les éléments structurants du dispositif. Ils organisent en effet l'accès aux ressources numériques et sont eux-mêmes subdivisés en souschapitres. Les entrées dans le corpus sont donc sémantiques et s'effectuent en premier lieu par l'affichage de la liste de documents nommés par leurs côtes d'archives. La collection numérique est ainsi livrée en son entier mais cette livraison ne se fait pas, en réalité, au fil des documents mais selon une série de thématiques. Celles-ci - «Plan de Bordeaux ", « Aménagement et urbanisme », « Port », «Équipements fonctionnels " et "Équipements de loisir " - définissent les objets architecturaux travaillés par Cyprien Alfred-Duprat. Par ailleurs, si l'aperçu du corpus via cette première organisation thématique propose une consultation linéaire, système de lecture permettant de prendre conscience rapidement et visuellement de la taille de celui-ci, les différents index gèrent ensuite l'accès aux substituts par une structure tabulaire (fig.2). L'accès aux archives est alors chronologique ("Index descripteur date »), géographique ( Index descripteur géographique »), monumental ("Index descripteur bâtiment ») ou par mots-clés ( Index descripteur mots outils »). Or, comme le note Cécile Tardy au sujet des représentations documentaires de collections d'objets muséaux sur le 
web : "Alors que la linéarité de la série accentue la distinction entre chaque objet - invitant à les regarder tour à tour -, le tableau réduit la singularité des parties - chaque objet - au profit de l'appréhension du tout - la collection" (Tardy, 2012 : 192). La cohabitation sur la même page des deux formes de mises en scène n'est pas sans entrainer, selon nous, une certaine confusion dans les possibilités de lecture des documents. Ainsi, le riche travail de description documentaire effectué par les experts patrimoniaux et réuni dans le tableau des «Index » reste sous exploité par les enseignants. Loin d'être rassurés par cette organisation documentaire parfaitement normée, ces derniers expriment ressentir l'éparpillement thématique du dispositif : "les sujets me paraissent trop larges, trop riches... un cadre plus resserré serait peut-être plus pertinent et plus efficace pour notre approche du corpus " [TDE], souligne par exemple un des professeurs. L'impression qui ressort des observations d'exploitation menées en classe, impression confirmée par le ressenti exprimé par les enseignant, est celle d'une appropriation désordonnée du corpus liée à une structuration hybride, entre description thématique et organisation archivistique.

\section{Une médiation documentaire qui met à distance le document}

Paradoxalement donc, l'appréhension cognitive des informations que contiennent ces ressources est rendue délicate par l'exhaustivité de l'indexation qui traite chaque document comme une unité fermée. Cette structuration se retrouve dans les modalités d'affichage du substitut numérique. Pour accéder aux documents, qu'il passe par la liste complète ou par les tableaux descripteurs, l'usager doit en effet cliquer sur la côte de l'archive dont la reproduction s'ouvre ensuite dans la même fenêtre en «l'écrasant ». L'utilisateur n'a donc aucune vision préalable du document qu'il va ensuite observer. De plus, l'espace de travail sur les images n'est pas rendu autonome puisque pour consulter d'autres ressources, l'utilisateur est contraint de revenir en arrière, retombant sur la page d'accueil du plan de classement. Il est par exemple impossible pour l'enseignant de créer un parcours de lecture des images fluide et indépendant de la structure générée par la page d'accueil. Ces allers-retours sont mal appréhendés par les enseignants qui regrettent la relégation au second plan des images, alors même que, numérisées en très haute résolution, leur affichage sur le TNI favorise la captation et l'attention des élèves sur une longue durée : « cette projection d'images très grandes et très lumineuses a un côté extrêmement esthétique, ce qui facilite la concentration... pendant ce temps les élèves ne feuillettent pas autre chose et leur curiosité est aiguisée... nous parlons de la même chose... ces projections fédèrent finalement le regard... » [CPA].

Aux termes de cette rapide description, plusieurs remarques peuvent être formulées quant aux mises en scène de ce regroupement d'images. On soulignera d'une part l'emploi d'un vocabulaire expert propre à la discipline archivistique, tels que index ou descripteurs qui ne sont pas des termes traditionnellement exploités par les enseignants destinataires du dispositif. Des expressions telles que "parcours" ou «thématiques " sont d'ailleurs plus généralement usitées par les sites internet de musées proposant aux enseignants des ressources et dossiers pédagogiques (Kerret et Guadet, 2014). L'absence totale d'images sur les pages d'accès de cette base iconographique ne cesse, d'autre part, d'étonner. Alors même que leur «beauté " est soulignée par l'animatrice du patrimoine lors de la visite de la Maison Cantonale et que la qualité de la numérisation est mise en avant par les différents acteurs du programme, celles-ci sont comme totalement oubliées par le système de lecture. En outre, les circulations hypertextuelles, multiples mais très hiérarchisées car organisées par les catégories et sous-catégories citées ci-dessus, ne favorisent pas la manipulation des images et donc l'exploitation de chaque unité documentaire. Cette configuration est due à la nature même du dispositif numérique créé à partir du détournement d'un logiciel professionnel de gestion documentaire. Le dispositif numérique n'est en définitive pensé que comme un système de transport et de conservation 
d'archives numériques. La discipline professionnelle archivistique reste de fait prégnante dans l'architecture de l'outil qui reproduit une certaine parenté avec les registres et classeurs de récolement clos sur eux-mêmes. "La page de présentation du corpus est fortement normée et relègue finalement l'image au second plan puisque comme en Archives, c'est la côte qui prime" [JCL] reconnait l'archiviste ayant programmé le système. Si, en tant qu'interface informatique traduisant l'accès aux ressources archivistiques sélectionnées, le dispositif matériel construit dans le cadre du programme "Cas d'école " peut être qualifié de médiation documentaire (Liquète et al., 2010), cette médiation numérique et documentaire est finalement plus organisationnelle que culturelle. Celle-ci autorise assurément l'accès à la ressource mais pas véritablement à son contenu. L'outil tel qu'il est actuellement pensé imprime dès lors une appréhension qui s'appuie sur les normes et techniques de description archivistiques. En limitant les possibilités de manipulation de ces ressources, en les éloignant par la surexposition de la côte au détriment de l'image, selon le système de nomination propre à la pratique des archivistes, en évacuant, enfin, la dimension esthétique de ces documents, ce système outillé ne favorise visiblement pas la mise en place d'une réelle médiation numérique culturelle.

\section{Conclusion}

Le champ des possibles pour la mise en place de nouveaux usages des ressources historiques numérisées est vaste, complexe et encore largement en construction. Les tentatives menées au sein de l'Éducation nationale représentent à cet égard un terrain d'expérimentation des médiations numériques intéressant (Inaudi, et Liautard, 2010). La politique particulière de l'Éducation artistique et culturelle sollicite notamment de nombreux acteurs qui dépassent le cadre institutionnel de l'Éducation Nationale et du Ministère de la Culture et de la Communication, réseau d'acteurs aux compétences documentaires et pédagogiques ancrées dans des pratiques de terrain plus traditionnelles. Cette multiplication des acteurs est a priori une richesse dans une politique de médiation culturelle et patrimoniale. L'exemple étudié ici montre toutefois combien il est difficile d'aboutir à un consensus autour de ce qui constitue l'essence même de la médiation, et a fortiori de la médiation numérique, l'appropriation. Alors que l'expérience menée par l'animatrice de la ville lors de la découverte de la Maison Cantonale est construite sur des thématiques sensibles selon une formule de transmission désormais bien maîtrisée par les acteurs de la médiation monumentale, la visite aux Archives et le dispositif numérique déroulent un discours qui met d'abord en scène la structure institutionnelle et son mode de travail. Le document, objet paradoxalement à l'origine du projet, est dès lors mis à distance du public. Dans le premier cas, la médiatrice déconstruit le monument en amenant progressivement les élèves vers sa complexité artistique en le confrontant à sa représentation documentaire qui, dès lors, devient surtout un objet de comparaison. Puis, en situation d'exploitation du dispositif numérique, le document s'éloigne et avec lui sa complexité informationnelle, tout comme aux Archives il demeure à l'abri des doigts impatients et maladroits des élèves.

Originaux protégés et intouchables aux Archives, image manipulable à la Maison Cantonale et substituts numériques en classe; les modes et contextes de lecture influencent la réception du document. Les logiques qui sous-tendent ces politiques pédagogiques également. Les pratiques, qu’il s'agisse de la description monumentale menée par l'animatrice en situation de visite guidée, comme de celles de conservation et de gestion documentaires assurées par les archivistes, sont ainsi promues par cette activité qui valorise une sorte de mémoire institutionnelle et professionnelle. Plus qu'une mémoire patrimoniale, c'est celle-ci qui s'intercale dans l'espace créé par l'éloignement des ressources iconographiques. Car finalement, cette expérience réanime l'opposition et la parenté qui 
unissent document et monument: «Là où le monument entretient reconnaissance et mémoire identitaire, le document engage des processus de connaissance et de savoir " (Merzeau, 1999, 51). Et c'est l'appropriation de ces connaissances et de ces savoirs qui demeurent difficiles à mettre en œuvre. Les professionnels des archives sont ainsi les détenteurs d'un cadre normé de description et de diffusion qui conditionne les possibilités d'appropriation et donc la mise en place d'une activité de médiation. Le dévoiement d'un système de classement numérique existant, en particulier, ne permet pas la construction d'une véritable médiation numérique qui favoriserait le dévoilement de la complexité documentaire en partant de la simplicité de l'objet document. La médiation, notamment lorsqu'elle est culturelle, semble finalement être une affaire d'interactions, qu'elles soient humaines ou numériques. Diffuser et décrire ne suffisent pas; reste à envisager des systèmes d'expérience cognitive aussi puissants que ce que peut vivre, par exemple, un sujet en situation de visite guidée mais aussi un joueur déambulant dans un univers virtuel.

\section{Références bibliographiques}

Both, Anne et Garcia, Guillaume (2014), « Le chercheur, l'archiviste et le webmaster : la polyphonie patrimoniale? Le cas BEQuali, banque d'enquêtes qualitatives en sciences sociales ", (353-364), in Dufrêne, Bernadette (dir.), Heritage and digital Humanities, Berlin : LIT.

Brun-Picard, Céline et Lallich-Boidin, Geneviève (2011), "L'accès au patrimoine écrit en ligne : analyse structurelle et réflexion prospective sur un cas français ", Les Enjeux de l'information et de la communication, [en ligne] http://lesenjeux.u-grenoble3.fr/2011/BrunPicard-Lallich/index.html

Casemajor-Lousteau, Nathalie (2013), «Valorisation du patrimoine photographique. Entre régime documentaire et régime artistique ", Culture et Musées, n²1, p. 43-63.

Chaumier, Serge et Mairesse, François (2013), La médiation culturelle, Paris : A. Colin.

Ciosi, Laure (2013), Étude sur la politique des publics et ses partenariats fonctionnels dans le réseau des archives municipales, départementales et régionales en France métropolitaine. Étude, Marseille : Transverscité.

Dufrêne, Bernadette, Ihadjadene, Madjid et Bruckmann, Denis (2013), Numérisation du patrimoine : quelles médiations? Quels accès ? Quelles cultures ?, Paris : Hermann.

Inaudi, Aude et Liautard, Dominique (2010), « De l'intérêt d'interroger les usages des ENT du point de vue de la médiation. Étude du dispositif CORRELYCE, Catalogue Ouvert Régional de Ressources Éditoriales pour les Lycées ", Les Enjeux de l'information et de la communication, [en lignel http://lesenjeux.u-grenoble3.fr/2010-dossier/Inaudi-Liautard/index.html

Jeanneret, Yves (2008), Penser la trivialité. Vol. 1 : La vie triviale des êtres culturels, Paris : HermèsLavoisier.

Jeanneret, Yves et Souchier, Emmanuël (2005), «L'énonciation éditoriale dans les écrits d'écran », Communication et langages, $\mathrm{n}^{\circ} 145$, p. 3-15.

Kerret, Gwenaëlle (de) et Guadet, Charlotte (2014), "Interface de consultation des ressources numériques: le cas des rubriques éducatives. Quelle posture du musée dans la relation 
pédagogique?»(225-242), in Dufrêne, Bernadette (dir.), Heritage and digital Humanities, Berlin : LIT.

Klein, Anne, et Lemay, Yvon (2014), « Les archives à l'ère de leur reproductibilité numérique » (3750), in La médiation numérique. Renouvellement et diversification des pratiques. Actes du colloque Documents numériques et société, Zagreb, 2013, Paris : de Boeck, ADBS.

Le Marec Joëlle (1993), "L'interactivité, rencontre entre visiteurs et concepteurs ", Publics et Musées, n³, p. 91-109.

Liquète, Vincent, Fabre, Isabelle et Gardiès, Cécile (2010), "Faut-il reconsidérer la médiation documentaire? ", Les Enjeux de l'information et de la communication, [en ligne] http://lesenjeux.ugrenoble3.fr/2010-dossier/Liquete-Fabre-Gardies/index.html

Merzeau, Louise (1999), « Du monument au document », Les cahier de médiologie, n7, p. 47-58.

Régimbeau, Gérard (2014), « Documents partagés », Documentaliste Sciences de l'information, vol. $51, \mathrm{n}^{\circ} 2$, p. $46-48$.

Sibers, Jean-François (2011), "Banque numérique du savoir en Aquitaine", Bulletin des bibliothèques de France, [en ligne] http://bbf.enssib.fr/consulter/bbf-2011-04-0050-010

Tardy, Cécile (2012), Représentations documentaires de l'exposition, Paris : Hermann.

Vatican, Agnès (2011), "Convergences et divergences entre archives et bibliothèques ", Bulletin des bibliothèques de France, [en ligne] http://bbf.enssib.fr/consulter/bbf-2011-04-0016-003

\section{Annexes iconographiques}

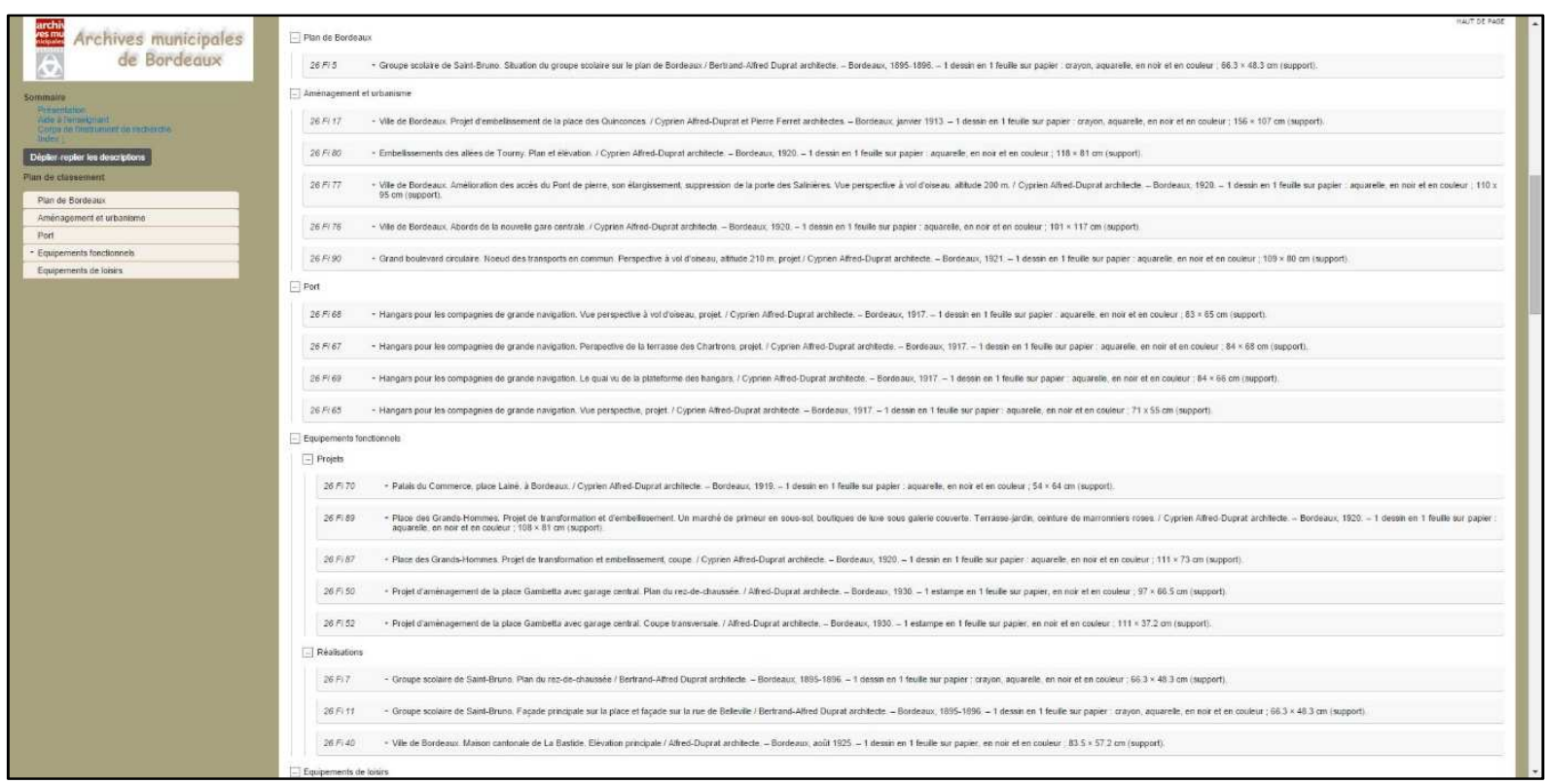

Fig. 1 - Structure d'organisation linéaire du corpus. Crédits Archives municipales de Bordeaux, «Cas d'école », 2011. 


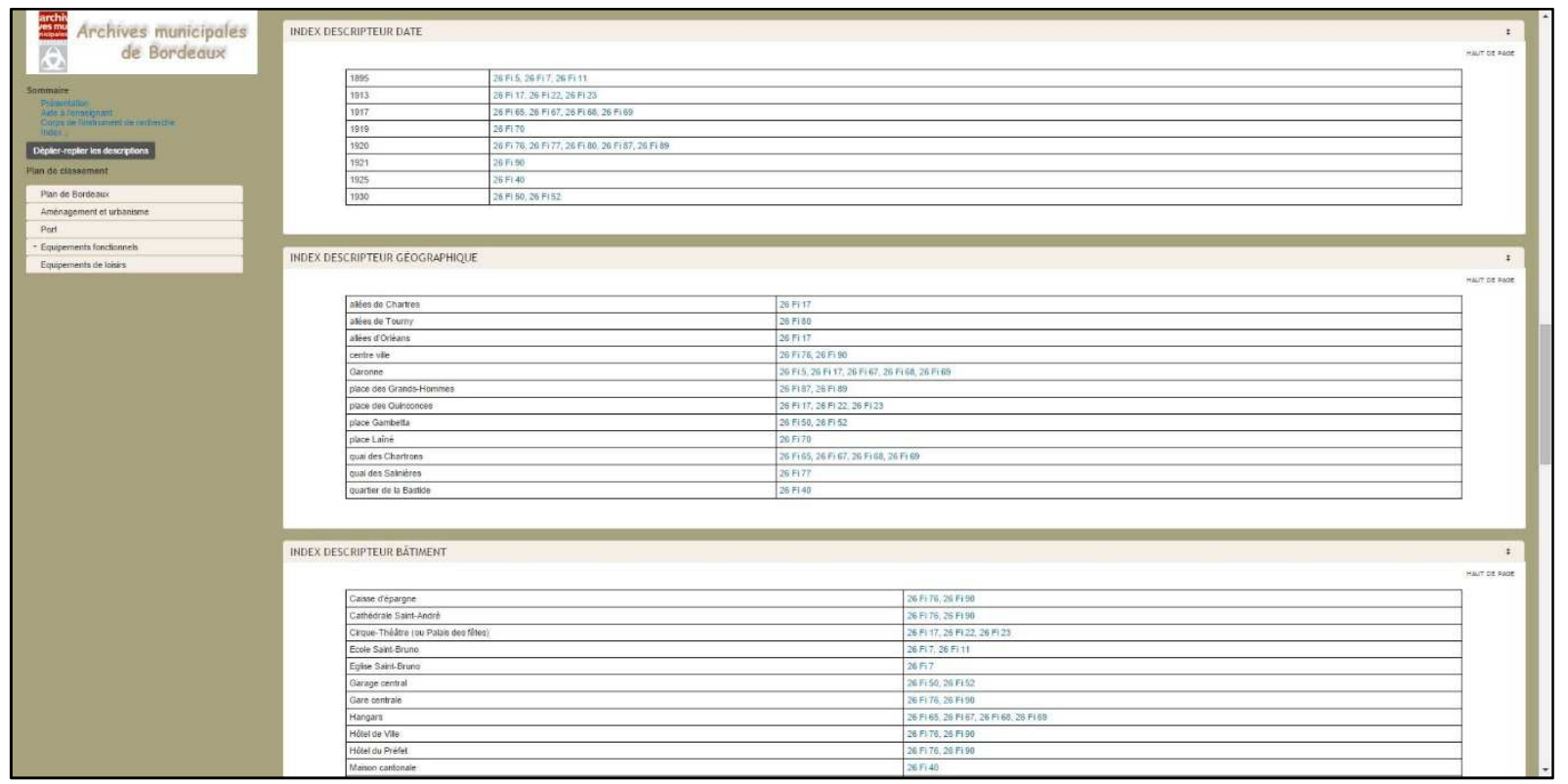

Fig. 2 - Structure d'organisation tabulaire du corpus. Crédits Archives municipales de Bordeaux, «Cas d'école », 2011. 